\title{
Végétation ligneuse du conservatoire botanique Michel Adanson de Mbour (Sénégal)
}

\author{
Richard Demba DIOP ${ }^{1 *}$, Mame Samba MBAYE ${ }^{1}$, César BASSENE ${ }^{3}$, Ibou DIOP ${ }^{1}$, \\ Oumar SARR ${ }^{2}$, Abdoul Aziz CAMARA ${ }^{1}$, Mame Thierno Aby SY ${ }^{4}$ et Kandioura NOBA ${ }^{1}$ \\ ${ }^{1}$ Laboratoire de Botanique et de Biodiversité, Département de Biologie Végétale, Faculté des Sciences et \\ Techniques, Université Cheikh Anta DIOP de Dakar, B.P.5005 Dakar-Fann, Sénégal. \\ ${ }^{2}$ Laboratoire d'Ecologie Végétale, Département de Biologie Végétale, Faculté des Sciences et Techniques, \\ Université Cheikh Anta DIOP de Dakar, B.P.5005 Dakar-Fann, Sénégal. \\ ${ }^{3}$ Section Productions Végétales et Agronomie, UFR des Sciences Agronomiques, de l'Aquaculture et des \\ Technologies Alimentaires, Université Gaston Berger de Saint Louis, BP. 234 Saint Louis, Sénégal. \\ ${ }^{4}$ ENDA-MADESAHEL, B. P. 29881 Mbour, Sénégal. \\ *Auteur correspondant ; E-mail: richarddiop2004@yahoo.fr; Tel: +221774378034
}

\section{RESUME}

La végétation du conservatoire botanique Michel Adanson de Mbour (CBMA), issue d'un processus de végétalisation naturelle et d'introduction d'espèces venant d'horizons diverses, est aujourd'hui menacée par un certain nombre de facteurs telle que l'érosion côtière. Or, une bonne gestion de cette végétation passe nécessairement par une meilleure connaissance de ses caractéristiques structurales. Cette étude a pour objectif de caractériser la structure de la végétation ligneuse du CBMA en vue d'une gestion durable. La méthode de relevés dendrométriques nous a permis de caractériser la végétation du CBMA. Les espèces ligneuses inventoriées sont au nombre de 23 réparties dans 21 genres et 16 familles. La densité, la surface terrière et le recouvrement des espèces sont assez élevés, entraînant une forte compétition entre les individus. Cela se traduit par un peuplement ligneux dominé par des individus de faible diamètre et de grande taille. Le peuplement ligneux est aussi caractérisé dans l'ensemble par une diversité moyenne et une distribution hétérogène des individus dans les parcelles. Il régénère assez bien avec la dominance de Leucaena leucocephala. Cette étude permet une meilleure connaissance de la végétation de la CBMA afin de mieux gérer ces ressources végétales. (C) 2019 International Formulae Group. All rights reserved

Mots clé : Végétation, flore ligneuse, Conservatoire botanique, ENDA- Madesahel, Mbour.

\section{Liguous vegetation of the botanical conservatory Michel Adanson of Mbour (Senegal)}

\begin{abstract}
The vegetation of the botanical conservatory Michel Adanson of Mbour (CBMA), resulting from a process of natural vegetation and introduction of species from various horizons, is today threatened by a number of factors such as erosion coastal. However, good management of this vegetation necessarily requires a better knowledge of its structural characteristics. The purpose of this study is to characterize the CBMA's
\end{abstract}


woody vegetation structure for sustainable management. The dendrometric survey method allowed us to characterize the CBMA vegetation. The woody species inventoried were 23 in 21 genera and 16 families. The density, the basal area and the recovery of the species were quite high causing a strong competition between the individuals. This results in a woody stand dominated by individuals of small diameter and size. The woody stand is also generally characterized by a medium diversity and heterogeneous distribution of individuals in the plots. It regenerates quite well with the dominance of Leucaena leucocephala. This study allows a better knowledge of the vegetation of the CBMA to better manage these plant resources.

(C) 2019 International Formulae Group. All rights reserved.

Keywords: Vegetation, woody flora, Botanical conservatory, ENDA- Madesahel, Mbour.

\section{INTRODUCTION}

La notion de développement durable cherche à harmoniser les nécessités en termes de développement économique et social et de conservation des ressources. Ces mots, développement et conservation, semblent antinomiques et pourtant, leur conciliation est aujourd'hui l'exigence minimale au regard de l'impact environnemental actuel du mode de vie humain (Ouedraogo et al., 2008). Les actions anthropiques sont responsables des changements les plus rapides des communautés végétales, même si le facteur climatique est le plus déterminant dans la distribution des espèces (Tremblay et al., 2002 ; Dessey, 2006). En effet, les principales causes de la déforestation et de la dégradation forestière sont liées à l'agriculture commerciale et de subsistance, à l'extraction du bois, à l'urbanisation et à la hausse émergente de plantations de biocarburants (PNUE, 2012).

La végétalisation est une action qui vise la reconstitution du couvert végétal à l'issue de travaux d'aménagement (Henry et al., 2011). Cette végétalisation se fait soit de façon naturelle ou de façon artificielle. Pour restaurer des boisements spontanés, il peut être intéressant de laisser évoluer les milieux concernés, qui seront peu à peu colonisés par des arbres et des arbustes indigènes adaptés et qui, à long terme, verront l'émergence de végétations forestières se reconstituant naturellement sans intervention humaine (Cornier et al., 2011 ; Henry et al., 2011). Lorsque la recolonisation ou la régénération naturelle forestière est difficile ou impossible, il est possible de "forcer" la dynamique naturelle en effectuant des plantations. Dans le cas de la végétalisation "artificielle", le matériel végétal provient d'une zone extérieure à celle des travaux. Cette solution est en général la plus utilisée en raison de la disponibilité des semences et des plants (Henry et al., 2011).

Dans une zone fortement anthropisée comme Mbour, les arbres ont plus de chance de survie dans une aire protégée qu'en dehors. Ainsi l'érection du conservatoire botanique Michel Adanson répond à ce problème de conservation durable d'espèces végétales et de maintien du peuplement végétale. Le site du conservatoire était jadis un espace cultivé, une zone de dunes à dominance de Maytenus senegalensis, qui a enregistré une introduction d'espèces nouvelles et connaît maintenant plus de vingt ans d'évolution en tant que formation naturelle enrichie (Mballo, 2011). La flore ligneuse se constitue progressivement en une végétation artificielle. Malgré cet enrichissement, elle connaît tout de même une certaine agression liée aussi bien à la péjoration climatique, qu'à des faits anthropiques. Et si rien n'est fait, cette végétation risque de disparaitre.

La végétation du conservatoire botanique Michel Adanson a déjà fait l'objet d'une étude (Mballo, 2011). Cependant elle était partielle car limitée seulement dans quatre parcelles sur seize (13 à 16). Or, pour une gestion durable, il est important de connaître la structure, la répartition spatiale et le potentiel de régénération de la végétation artificielle. Ainsi, le présent travail envisage de caractériser la diversité compositionnelle et structurale de la végétation ligneuse du conservatoire botanique Michel Adanson en vue d'une gestion durable. 


\section{MATERIEL ET METHODES \\ Présentation du site d'étude}

Le conservatoire botanique Michel Adanson (Figure 1) $\left(14^{\circ} 25^{\prime} 14.40^{\prime \prime} \mathrm{N}\right.$ et $\left.16^{\circ} 59^{\prime} 20.19^{\prime \prime} \mathrm{O}\right)$ est localisé à Mbour. Il s'étend sur 4 hectares autour du Centre d'Application au Développement Intégré (CADI) qui a une superficie de 4,97 ha. Il est situé à $82 \mathrm{~km}$ au sud de Dakar, dans un quartier de la ville de Mbour, dénommé Grand Mbour, en bordure de mer. La commune de Mbour couvre une superficie de 1725 ha. Chef-lieu du département qui porte le même nom, elle est localisée entre le Cap-Vert et la pointe de Sangomar en demi-cuvette fermée à l'Ouest par l'océan Atlantique. Cette collectivité est limitée au nord, à l'Est et au Sud par la communauté rurale de Malicounda. Sa localisation en bordure de mer entre le Cap-Vert et la Pointe de Sangomar lui confère de nombreux atouts (pèche et tourisme) qui expliquent son poids démographique (Tine, 2009). Les sols sont majoritairement de type Dior dans le département (plus de 50\%), avec une partie latéritique dans la communauté rurale de Diass et à Sindia. Une partie Deckdior à caractéristique plus prononcée de Deck est également notable à Mbodjène (communauté rurale de Ngéniène). Le cumul annuel des pluies des années 2008 et 2009 fut respectivement $605,4 \mathrm{~mm}$, étalé sur 60 jours et, $673,2 \mathrm{~mm}$ pour 37 jours. Pour l'année 2010, ce cumul fut $907,6 \mathrm{~mm}$, pour 56 jours de pluies. Faute d'habitats, la faune sauvage est très peu représentée dans le département, même s'il faut reconnaître les efforts de réintroduction en grande et petite faune, dans la réserve de Bandia qui est un véritable exemple de conservation de la biodiversité faunique et floristique dans le département (Tine, 2009).

\section{Etude de la végétation}

Pour caractériser la diversité végétale, nous allons d'abord définir le terme diversité en empruntant la définition de biodiversité de Crow et al. (1994), qui a distingué trois groupes de diversité; à savoir la diversité compositionnelle, la diversité structurale et la diversité fonctionnelle (Diatta et al., 2009).
La diversité compositionnelle considère le nombre de taxons présent dans un espace ; c'est la richesse spécifique lorsqu'il s'agit des taxons végétaux. La diversité structurale peut être caractérisée par la distribution horizontale et ou verticale des plantes, par leur distribution en classe de taille. La diversité fonctionnelle s'intéresse au processus écologique qui se déroule dans l'écosystème considéré. Dans le cadre de cette étude, nous allons établir la diversité compositionnelle et la diversité structurale.

Les méthodes d'inventaire se différencient en deux catégories: les inventaires pied par pied, où tous les arbres sont inventoriés, et les inventaires statistiques, où seule une partie de la forêt est inventoriée. La deuxième méthode a été utilisée car même si la superficie de notre site n'est pas assez grande (environ 5ha), la densité de la végétation est très importante et nos moyens très limités.

Un échantillonnage par grappe a été réalisé : les placettes d'échantillonnage sont regroupées en grappes de forme fixe comportant 5 sous placettes, ce qui limite les déplacements (Picard, 2006).

L'échantillonnage a utilisé une placette circulaire de $100 \mathrm{~m}$ de rayon $(3,14$ ha) placée au milieu du conservatoire à l'aide du GPS (Figure 2). A l'intérieur de cette placette, nous avons également placé au niveau des quatre côtés et au milieu une sous placette circulaire de $20 \mathrm{~m}$ de rayon soit $0.125 \mathrm{ha}$. A l'intérieur de chaque sous placette circulaire, nous avons placé des sous placettes carrés de $10 \mathrm{~m}$ de côté. La surface totale inventoriée est égale à 0.6 ha.

$\mathrm{Au}$ niveau de chaque sous placette circulaire, les observations ont consisté à recenser tous les individus ligneux dont le diamètre à hauteur de poitrine (D.B.H.) supérieur $10 \mathrm{~cm}$. Ceci a permis d'établir la liste de la flore ligneuse.

Pour chaque individu rencontré, les paramètres suivant seront mesurés :

-la hauteur pour établir la structure verticale du peuplement ;

-la distance entre deux individus en utilisant la méthode du plus proche individu 
(PPI) afin d'établir la densité théorique, et ainsi la distribution des ligneux;

-le diamètre à la base du tronc à $30 \mathrm{~cm}$ $\mathrm{du}$ sol, pour estimer la surface terrière et étudier la répartition des individus ligneux suivant les classes de diamètre. Pour les individus multicaules, seul le plus gros diamètre est pris en compte.

-le diamètre du houppier des arbres, pour évaluer le recouvrement aérien.

A l'intérieur de chaque sous placette carré, tous les individus dont le dbh est inférieur à $10 \mathrm{~cm}$ sont systématiquement considérés comme faisant partie de la régénération et sont comptés.

\section{Traitement de données}

Les données sur la végétation ligneuse ont été traitées avec le logiciel Excel et Xlstat 2015. Le traitement a essentiellement consisté au calcul des paramètres comme la fréquence, la densité, la surface terrière, le recouvrement, utilisés pour caractériser la végétation ligneuse :

\section{Fréquence de présence}

La fréquence est le nombre de fois qu'une espèce apparaît dans une série d'échantillonnage. La fréquence de présence à une espèce d'une communauté végétale donnée s'obtient par la formule de RobertsPichette et Gillespie (2002):

$F=\frac{N r i}{N r} \times 100$

$\mathrm{F}=$ fréquence de présence en pourcentage $(\%)$ $\mathrm{Nri}=$ nombre de relevés où l'on retrouve l'espèce i

$\mathrm{Nr}=$ nombre total de relevés

\section{Densité des tiges à l'hectare}

La densité d'une espèce correspond au nombre de pieds appartenant à l'espèce par unité de surface. Elle est exprimée en nombre d'individus par hectare (n.ha ${ }^{-1}$.). Cette densité a été déterminée de deux manières différentes :

Densité réelle

Cette densité étant donnée par le rapport de l'effectif total de l'espèce dans l'échantillon par la surface de cet échantillon :

$$
\text { Dob. }=\frac{N i}{S}
$$

Avec $D_{o b}=$ Densité observée ;

$\mathrm{Ni}=$ Effectif total de l'espèce $i$ dans l'échantillon considéré et

$S=$ surface de l'échantillon en ha.

\section{Densité théorique}

Avec les distances moyennes entre arbres, cette densité est obtenue à partir de la formule suivante :

$D_{t h .}=\left(\frac{100}{d m}\right)^{2}$

Avec $\boldsymbol{D}_{t h .}=$ Densité théorique ;

$\boldsymbol{d}_{\boldsymbol{m}}=$ distance moyenne en mètre $(\mathrm{m})$.

\section{Surface terrière des bois vivants}

$\mathrm{La}$ surface terrière est une grandeur couramment utilisée par les forestiers. Il s'agit de la somme des surfaces des sections des arbres vivants à $1,30 \mathrm{~m}$ exprimée en $\mathrm{m}^{2}$. Elle indique la quantité de matériel vivant en présence et la compétition entre les arbres. Cependant, seuls les arbres dont le diamètre à $1,30 \mathrm{~m}$ est supérieur à $20 \mathrm{~cm}$ sont concernés. Pour obtenir la surface terrière des bois vivants totale, on multiplie les contributions des arbres à la densité du peuplement par la surface de la section du tronc à $1,30 \mathrm{~m}$, exprimée en $\mathrm{m}^{2} / \mathrm{ha}$.

$$
S t=\frac{\pi\left(\frac{d o, 3}{2}\right)^{2}}{S_{E}}
$$

Avec $S_{t}=$ surface terrière;

$\boldsymbol{d}_{\boldsymbol{0}, 3}=$ diamètre en $\mathrm{m}$ du tronc à $0,3 \mathrm{~m}$ du sol, $S_{E}=$ surface de l'échantillon considéré en ha.

\section{Recouvrement aérien}

Le couvert aérien est la projection verticale de la surface de la couronne de l'arbre au sol. Il indique la portion du sol couverte par le feuillage de l'arbre (RobertsPichette et Gillespie, 2002 ; Badji et al., 2013). Il s'exprime en $\left(\mathrm{m}^{2}\right.$.ha. $\left.{ }^{-1}\right)$ et est calculé avec la moyenne des diamètres nord-sud et est-ouest du houppier des arbres avec la formule ci-dessous : 
$G=\frac{\pi\left(\frac{d m h}{2}\right)^{2}}{S_{E}}$

où $G=$ couvert aérien ;

$d_{m h}=$ diamètre moyen du houppier en $\mathrm{m}$, qui est égal à la moitié de la somme des diamètres Nord-Sud et Est-Ouest ;

$S=$ surface de l'échantillon considéré en ha.

\section{Indices de diversité biologique \\ Indice de Shannon}

Pour caractériser la diversité floristique des différents secteurs du conservatoire botanique, nous avons utilisé l'indice de Shannon $(\mathrm{H})$ car c'est l'un des meilleurs estimateurs de la diversité biologique (Magurran, 2005). Cet indice est utilisé comme mesure d'hétérogénéité et prend en compte la régularité d'abondance des espèces (Peet, 1974). La diversité est faible lorsque $\mathrm{H}$ est inférieur à 3 bits, moyenne si $\mathrm{H}$ est compris entre 3 et 4 puis élevé quand $\mathrm{H}$ est supérieur ou égal à 4 bits (Legendre et Legendre, 1984 ; Frontier et Pichod-Viale, 1995 ; Adjaka et al., 2013). L'indice de Shannon est calculé par la formule suivante :

$$
\boldsymbol{H}=-\sum_{i=1}^{S} p i \cdot \log 2(p i)
$$

où $H=$ Indice de Shannon ;

$p_{i}=$ abondance proportionnelle ou pourcentage d'importance de l'espèce, $\mathrm{pi}=\mathrm{ni} / \mathrm{N}$;

$S=$ Nombre total d'espèce;

$\mathrm{ni}=$ nombres d'individus d'une espèce dans l'échantillon ;

$\mathrm{N}=$ nombres total d'individus de toutes les espèces dans l'échantillon.

\section{Indice d'équitabilité de Pielou}

L'équitabilité de Pielou (J) traduit la manière dont les individus sont distribués à travers les espèces. Elle est maximale si les individus sont répartis de la même manière à travers les espèces. Elle varie de 0 (une espèce a une très forte abondance) à 1 (toutes les espèces ont la même importance) (Adjakpa et al., 2013). L'indice d'équitabilté de Piélou (J)est calculé par la formule suivante :

$$
J=\frac{H}{H \max }
$$

Où $J=$ Indice d'équitabilité ;

Hmax $=\log _{2}(\mathrm{~S})$;

$S=$ nombre total d'éspeces.

L'indice de diversité de Shannon et celui d'équitabilité sont deux mesures de la diversité spécifique parmi les plus connues. Ils sont calculés à partir de fréquences des espèces rencontrées. Ces indices qui permettent de caractériser la diversité floristique d'une collection de relevés, constituent de bons points de repère et ont un fort sens opérationnel dans les stratégies de réhabilitation d'écosystèmes dégradés par le maintien ou l'amélioration d'une productivité à long terme (Djego et al., 2012).

\section{Taux de régénération}

La capacité de régénération du site a été appréciée par le calcul du taux de régénération du peuplement (TRP). Le taux de régénération du peuplement est donné par le rapport en pourcentage entre l'effectif total des jeunes plants et l'effectif total du peuplement (Poupon, 1980).

$$
T R P=\frac{\text { Effectif total des jeunes plants }}{\text { Effectif total du peuplement }} \times 100
$$

\section{Indice spécifique de régénération}

L'Indice spécifique de régénération est quant à elle obtenue à partir du rapport en pourcentage entre l'effectif des jeunes plants d'une espèce et l'effectif total des jeunes plants dénombrés (Akpo \& Grouzis, 1996)

$$
I S R=\frac{\text { Effectif des jeunes plants d' une espèce }}{\text { Effectif total des jeunes plants }} \times 100
$$




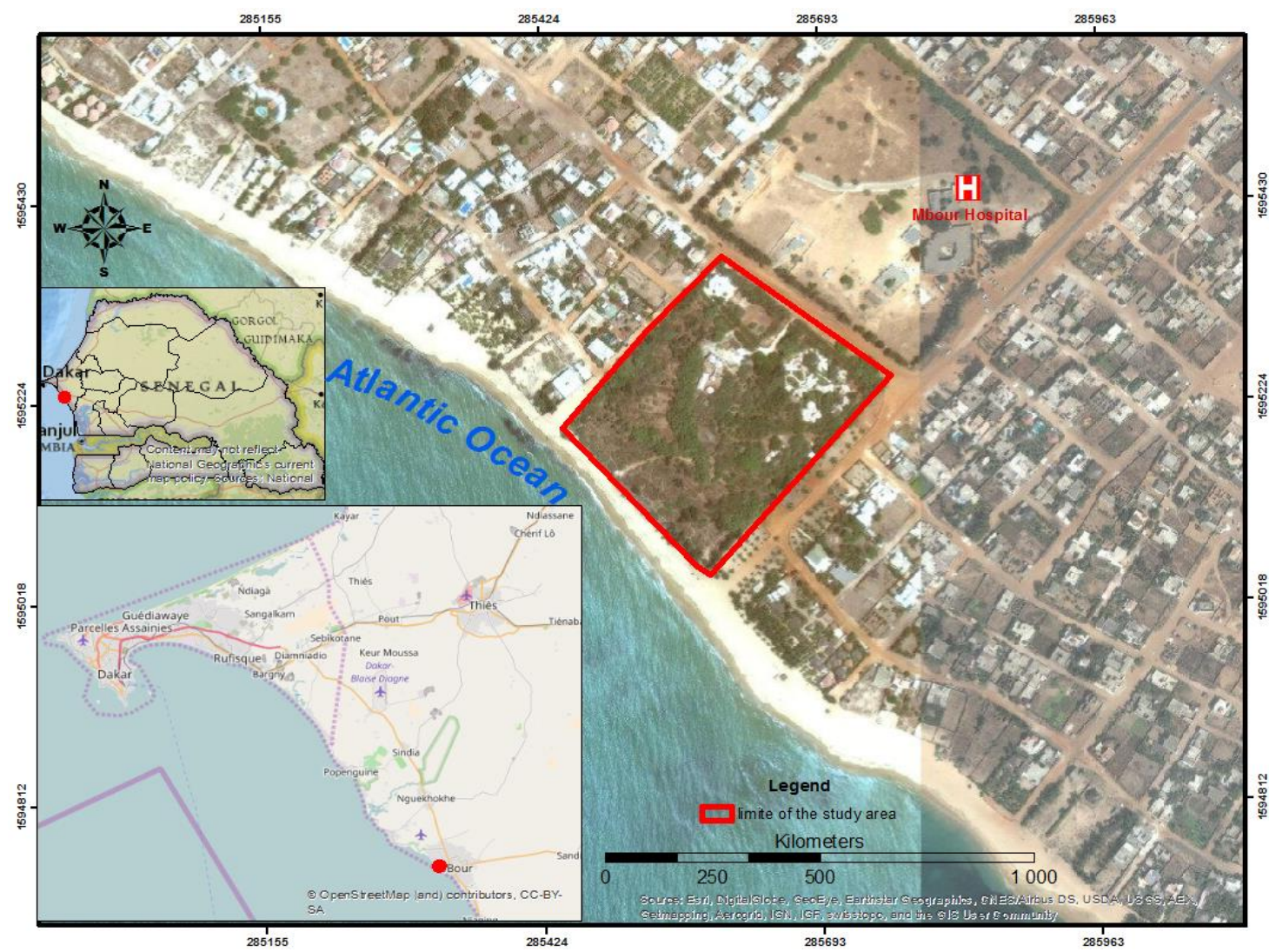

Figure 1: Situation géographique de la commune de Mbour et du conservatoire.

\section{RESULTATS \\ Caractéristiques de la végétation ligneuse La flore ligneuse}

La flore d'un milieu est définie par sa composition taxonomique (espèces, genres, familles). Nous avons recensé dans les 5 sous placettes d'inventaire 193 individus ligneux (d.b.h $\geq 10 \mathrm{~cm}$ ) réparties en 23 espèces, 21 genres et 16 familles (Tableau 1 ).

Sur le plan générique, la famille la mieux représentée est celle des Fabaceae (6 genres). Toutes les autres familles ne sont représentées que par un seul genre. Parmi les genres, Acacia (2 espèces), Terminalia (2 espèces) sont les mieux représentés. Tous les autres genres renferment une seule espèce chacun.

\section{Fréquence de présence}

Les espèces ligneuses les plus fréquentes sont Leucaena leucocephala (100\%), Prosopis juliflora (60\%), Kigali africana $(60 \%)$. Elles sont présentes dans plus de la moitié des relevés.

\section{Structures démographiques de la végétation \\ Densités}

La densité observée ou densité réelle (le nombre d'individus par hectare) et la densité théorique (à partir de la distance moyenne entre les arbres) ont été successivement examinées. La densité réelle est de 322 individus/ha (Tableau 2). Elle a beaucoup varié entre les espèces les plus représentées (Leucaena leucocephala 155 arbres/ha, Casuarina equisetifolia 45arbres/ha, Balanites aegyptiaca 18 individus/ha et Melaleuca leucadendron 17 arbres/ha) et les autres espèces qui ont une densité inférieure à 15 arbres/ha.

La distance moyenne entre deux individus est de 3,52 m, avec un coefficient de variation de 49,2\% la densité théorique est alors de 807 individus à l'hectare soit plus 2 fois la densité observée. 


\section{Recouvrements}

Dans ce paragraphe, nous avons présenté successivement le recouvrement aérien et la surface terrière (recouvrement basal). Le couvert ligneux (aérien) total est de $5521,14 \mathrm{~m}^{2} /$ ha (Tableau 2 colonne 5), soit 92 $\%$ de la surface totale inventoriée. Il est largement dominé par Leucaena leucocephala avec 2369,59 $\mathrm{m}^{2} /$ ha soit $48,19 \%$ du recouvrement total. Cette espèce est représentée par des arbres à grandes cimes. Elle est suivie d'Acacia nilotica (722,85 $\left.\mathrm{m}^{2} / \mathrm{ha}\right)$, de Casuarina equisetifolia (511,22 $\left.\mathrm{m}^{2} / \mathrm{ha}\right)$ et de Balanites aegyptiaca $(320,39$ $\mathrm{m}^{2} / \mathrm{ha}$ ).

La surface terrière est la surface de la section transversale du tronc de l'arbre à $30 \mathrm{~cm}$ du sol. Elle est exprimée en $\mathrm{m}^{2} /$ ha. Elle est de $11,77 \mathrm{~m}^{2} /$ ha pour l'ensemble du peuplement inventorié, soit $0,20 \%$ de la surface totale inventoriée. Leucaena leucocephala est largement dominante $\left(3,76 \mathrm{~m}^{2} / \mathrm{ha}\right)$; elle a été suivie par Casuarina equisetifolia (1,42 $\left.\mathrm{m}^{2} / \mathrm{ha}\right)$, Acacia nilotica $\left(0,98 \mathrm{~m}^{2} / \mathrm{ha}\right)$, Balanites aegyptiaca $\left(0,97 \mathrm{~m}^{2} / \mathrm{ha}\right)$. Ces quatre espèces représentent $60,57 \%$ des valeurs de la surface terrière totale.

\section{Structure du peuplement \\ Répartition selon la hauteur}

La Figure 2 représente la distribution $\mathrm{du}$ peuplement et des espèces les plus fréquemment retrouvées dans le conservatoire selon la hauteur.

La distribution du peuplement (Figure 3A) par son aspect bimodal fait apparaître deux pics. Le premier est situé dans la classe 5,5-7,5m. Ce groupe est celui des individus dont la hauteur est inférieure à 9,5 m et correspond à une forte proportion d'individus de la strate arbustive avec la moitié des individus $(50,26 \%)$. Le deuxième pic situé dans la classe $9,5-11,5 \mathrm{~m}$ fait partie des individus dont la hauteur est supérieure à $9,5 \mathrm{~m}$. Les grands arbres sont très nombreux et représentent près de la moitié des individus (49,74\%).

La structure de la population de Leucaena leucocephala (Figure 3B) montre un modèle de distribution de type exponentiel croissant et illustre le modèle unimodal. Elle présente un pic dans la classe 9,5-11m de hauteur avec 60,22\% des individus. Certains individus dont la classe de hauteur est comprise entre 5,5-7,5 m sont faiblement représentés. Par contre, il n'existe pratiquement pas d'individu dont la hauteur est supérieure à $11,5 \mathrm{~m}$.

La population de Casuarina equisetifolia (Figure 3B) n'est constituée pratiquement que par des individus de la strate arborée (11,5-15,5 m). Elle représente 92,6\% de la population. La strate arbustive est faiblement représentée.

La structure de la population de Balanites aegyptiaca (Figure 3B) caractérisée par une distribution de type log normal à aspect unimodal aussi et montre un pic situé dans la classe $5,5-7,5 \mathrm{~m}$ avec $60 \%$ des individus. Tous les individus de Balanites aegyptiaca ont une hauteur inférieure à $11,5 \mathrm{~m}$. Ceci démontre que cette espèce constituerait l'essentielle de la strate arbustive.

\section{Répartition selon la grosseur}

La Figure 4 représente la distribution $\mathrm{du}$ peuplement et des espèces les plus fréquemment retrouvées dans la réserve selon le diamètre.

La même amplitude des classes de diamètre $(5 \mathrm{~cm})$ a été prise pour toutes les espèces. La Figure 4A illustre les résultats relatifs aux variations de fréquences du peuplement ligneux. L'histogramme du peuplement présente un modèle de distribution de type exponentiel décroissant caractérisé par la dominance des individus de faible diamètre. Les classes de $10-25 \mathrm{~cm}$ renferment $87,56 \%$ des individus et $67,87 \%$ de ces individus sont représentés uniquement dans la classe de $10-20 \mathrm{~cm}$. Au niveau des classes supérieures ou égales à $25 \mathrm{~cm}$, les individus sont faiblement représentés avec une fréquence de $20,73 \%$. Il en est même pour la population de Leucaena leucocephala (Figure 4B) qui est composée en majorité d'arbustes dont le diamètre est compris entre 10 et $20 \mathrm{~cm}$ et représentant $80,64 \%$ des individus. Ce groupe d'individus présente un pic entre 10 $15 \mathrm{~cm}$ avec $61,28 \%$ des individus. Les individus dont le diamètre est supérieur ou égale à $20 \mathrm{~cm}$ sont faiblement représentés avec $15,78 \%$ des individus. 
La structure de Casuarina equisetifolia et de Balanites aegyptiaca montre une distribution de type log normal avec quelques différences. L'histogramme de structure de Casuarina equisetifolia montre que la totalité des individus ont une classe de diamètre comprise entre 10-30 $\mathrm{cm}$. Toutefois la classe $10-15 \mathrm{~cm}$ regroupe près de la moitié des individus (44,44\%). Le diamètre des Casuarina ne dépasse pas $30 \mathrm{~cm}$.

De par l'aspect bimodal, la distribution de la population de Balanites aegyptiaca montre deux groupes d'individus. Le premier pic est situé dans la classe $15-25 \mathrm{~cm}$ alors que le deuxième dans la classe de $45-50 \mathrm{~cm}$. Le premier groupe qui a un diamètre inférieur à $30 \mathrm{~cm}$ représente $80 \%$ des individus essentiellement des jeunes. Le deuxième groupe, d'un diamètre supérieur ou égal à 45 $\mathrm{cm}$ représente $20 \%$ des individus qui sont adultes.

Les histogrammes de distribution selon la hauteur et les histogrammes de distribution selon la circonférence sont en phases parce que dans les classes inférieures se trouvent les arbustes comme Balanites aegyptiaca et dans les classes supérieures, les arbres à l'image de Casuarina equisetifolia et Leucaena leucocephala.

\section{Répartition spatiale}

La matrice de 23 espèces fois 5 relevés a été soumise à l'analyse factorielle de correspondance pour voir la dispersion des plantes ligneuses inventoriées dans les différents secteurs du conservatoire botanique Michel Adanson (Est, Ouest, Nord, Sud et Centre). Les points lignes et les points colonnes désignent respectivement les espèces (ou variables) et les relevés (ou observations).

La contribution des secteurs et des plantes ligneuses est mesurée par leurs valeurs propres. Ainsi, le poids relatif des secteurs a varié de $0,070 \%$ à $0,140 \%$ et celui des espèces ligueuses inventoriées dans ces secteurs de $0,015 \%$ à $0,203 \%$. Les trois secteurs à forte contribution, c'est-à-dire présentant des

inerties supérieures à la moyenne sont les secteurs Nord, Sud et Est tandis que les espèces ligneuses sont Leucaena leucocephala, Casuarina equisetifolia, Melaleuca leucadendron, Balanites aegyptiaca, Moringa oleifera et Prosopis juliflora.

L'information contenue dans le tableau de données a été de 0,28 bits et projetée essentiellement au niveau de quatre premiers axes factoriels (Tableau 3). Les axes F1 et F2 ont respectivement $37,01 \%$ et $27,95 \%$ de l'information, soit $64,96 \%$ portés par le couple F1-F2. L'écart est fort $(9,06 \%)$ entre les axes F1 et F2. Donc les inerties des deux axes sont éloignées l'une de l'autre et semblent indiquer une dispersion hétérogène des plantes dans les différentes parcelles du conservatoire.

Le nombre d'axes factoriels est égal à la valeur du plus petit nombre des colonnes et lignes du tableau. Le traitement a extrait 4 axes factoriels. Les 2 premiers axes factoriels $(\mathrm{F} 1$ et $\mathrm{F} 2)$ totalisent près de $65 \%$ de l'information. L'analyse peut donc être faite sur ceux-ci.

L'exploitation de la Figure 5 nous a permis d'identifier 3 groupes distincts dans le conservatoire botanique Michel Adanson de Mbour. Le groupe 1 contient les espèces rares du conservatoire c'est-à-dire les moins fréquents souvent représentés par 1seul pieds. Ce sont des espèces qui sont sélectionnées par leur valeur thérapeutique et plantées dans l'enceinte même de l'ISED. On peut citer Mangifera indica, Manilkara zapota, Terminalia mantaly, Vernonia senegalensis, Adansonia digitata, Cocos nucifera, Gmelina arborea, Crataeva religiosa...

Le groupe 2 renferme des espèces situées à l'ouest du conservatoire en allant vers la mer et formant des groupements plus ou moins denses. C'est le cas de Prosopis juliflora qui forme la haie de certaines pistes du conservatoire, de Melaleuca leucadendron et de Tamarindus indica. Les espèces dominantes au Sud et au Centre du site (Acacia nilotica, Acacia tortilis, Commifora africana et Peltophorum pterocarpum) forment le groupe 3 .

\section{La diversité du conservatoire botanique}

Dans le conservatoire botanique, l'indice de Shannon est égal à 2 bits et celui d'équitabilité de Pielou est égal à 0,64 . Ces indices varient d'un secteur à un autre, ce qui a permis d'apprécier la différence de diversité 
existant entre les secteurs (Figure 6). La zone Est qui inclut l'ISED a un indice d'équitabilité de Pielou assez élevée $(0,63)$ donc c'est une zone de distribution assez homogène et régulière des plantes. Les espèces ont à peu près la même importance car constituées pour la plupart de pied unique. Son indice de Shannon est le plus grand $(2,26)$ par conséquent c'est une zone de diversité biologique assez élevée. Les zones Sud, Centre, Nord et Ouest ont des valeurs d'indices d'équitabilité faibles comprises entre 0,20 et 0,40 . Ce sont des zones caractérisées par la dominance d'un petit nombre d'espèces telles que Leucaena leucoceplela, Melaleuca lecadendron, et Balanites aegyptiaca. Leurs indices de Shannon sont également faibles car compris entre 0,80 et 1,44 . Ainsi, la diversité biologique dans ces zones est très faible malgré le nombre d'individus élevé.

\section{La régénération du peuplement}

Au niveau des 5 sous-placettes carrées, tous les individus dont le diamètre à hauteur de poitrine (soit à $1,30 \mathrm{~cm}$ du sol) est inférieur à $10 \mathrm{~cm}$ sont considérés comme jeunes plants. Au total, 183 jeunes plants, soit $48,67 \%$ de l'effectif du peuplement ont été recensés. La densité est de 3660 jeunes plants à l'hectare (Tableau 4).

La Figure 7 montre le taux de régénération du peuplement et l'indice spécifique de régénération des 3 espèces ligneuses les plus fréquentes du conservatoire. Leucaena leucocephala a enregistré l'indice de régénération le plus élevé $(50,82 \%)$, suivie de Maytenus senegalensis $(9,28 \%)$ et de Azadirachta indica $(8,20 \%)$.

Tableau 1: Liste des espèces ligneuses inventoriées et leur fréquence de présence.

\begin{tabular}{|c|c|c|c|}
\hline Familles & Genres & Espèces & $\begin{array}{l}\text { Fréquence de } \\
\text { présence }(\%)\end{array}$ \\
\hline ANACARDIACEAE (D) & Mangifera & Mangifera indica $\mathrm{L}$. & 20 \\
\hline ASRERACEAE (D) & $\begin{array}{l}\text { Gymnanthemu } \\
\mathrm{m}\end{array}$ & $\begin{array}{l}\text { Gymnanthemum coloratum (Willd.) } \\
\text { Rob. \& Kahn }\end{array}$ & 20 \\
\hline BIGNONIACEAE (D) & Kigelia & Kigelia africana (Lam.) Benth & 20 \\
\hline BURSERARACEAE (D) & Commiphora & Commiphora africana (A. Rich.) Engl. & 60 \\
\hline CAPPARIDACEAE (D) & Crataeva & Crataeva adansonii DC. & 20 \\
\hline CASUARINACEAE (D) & Casuarina & Casuarina equisetifolia Forst. & 20 \\
\hline \multirow{2}{*}{ COMBRETACEAE (D) } & \multirow{2}{*}{ Terminalia } & Terminalia catappa $\mathrm{L}$ & 40 \\
\hline & & Terminalia mantaly H. Perrier. & 40 \\
\hline \multirow{7}{*}{ FABACEAE (D) } & Bauhinia & Bauhinia rufescens Lam. & 20 \\
\hline & Peltophorum & $\begin{array}{l}\text { Peltophorum pterocarpum (DC) K. } \\
\text { Heyne }\end{array}$ & 40 \\
\hline & Tamarindus & Tamarindus indica $\mathrm{L}$. & 20 \\
\hline & \multirow{2}{*}{ Acacia } & Acacia nilotica (L.) Willd. ex Del. & 40 \\
\hline & & Acacia tortilis var. raddiana & 20 \\
\hline & Leucaena & Leucaena leucocephala (Lam.) de Wit & 20 \\
\hline & Prosopis & Prosopis juliflora (Sw.) DC. & 100 \\
\hline LAMIACEAE (D) & Gmelina & Gmelina arborea Roxb. & 60 \\
\hline MALVACEAE (D) & Adansonia & Adansonia digitata $\mathrm{L}$. & 20 \\
\hline MORINGACEAE (D) & Moringa & Moringa oleifera Lam. & 20 \\
\hline MYRTACEAE (D) & Melaleuca & Melaleuca leucadendron L. & 40 \\
\hline PALMAE (M) & Cocos & Cocos nucifera $\mathrm{L}$ & 20 \\
\hline SAPINDACEAE (D) & Lepisanthes & $\begin{array}{l}\text { Lepisanthes senegalensis (Juss. ex } \\
\text { Poiret) }\end{array}$ & 20 \\
\hline SAPOTACEAE (D) & Manilkara & Manilkara zapota (L.) P. Royen. & 20 \\
\hline ZYGOPHYLLACEAE (D) & Balanites & Balanites aegyptiaca (L.) Del. & 40 \\
\hline
\end{tabular}


Tableau 2 : Caractéristiques des espèces ligneuses.

\begin{tabular}{lcccc}
\hline Espèces & Effectifs & Dens. (ind. /ha) & $\begin{array}{c}\text { Rec. } \\
\left(\mathrm{m}^{2} / \mathrm{ha}\right)\end{array}$ & $\begin{array}{c}\text { Surf. Ter. } \\
\left(\mathrm{m}^{2} / \mathrm{ha}\right)\end{array}$ \\
\hline Acacia nilotica (L.) Willd. ex Del. & 7 & 12 & 722,85 & 0,98 \\
Acacia tortilis var. raddiana & 2 & 3 & 203,40 & 0,38 \\
Adansonia digitata L. & 1 & 2 & 63,59 & 0,91 \\
Balanites aegyptiaca (L.) Del. & 11 & 18 & 320,39 & 0,97 \\
Bauhinia rufescens Lam. & 5 & 8 & 70,29 & 0,11 \\
Casuarina equisetifolia Forst. & 27 & 45 & 511,22 & 1,42 \\
Cocos nucifera L. & 2 & 3 & 85,70 & 0,20 \\
Commiphora africana (A. Rich.) Engl. & 1 & 2 & 19,63 & 0,02 \\
Crataeva adansonii DC. & 1 & 2 & 7,07 & 0,03 \\
Gmelina arborea Roxb. & 1 & 2 & 24,18 & 0,03 \\
Gymnanthemum coloratum (Willd.) Rob. \& Kahn & 1 & 2 & 12,62 & 0,10 \\
Kigelia africana (Lam.) Benth & 3 & 5 & 80,78 & 0,22 \\
Lepisanthes senegalensis (Juss. ex Poiret) & 1 & 2 & 38,47 & 0,16 \\
Leucaena leucocephala (Lam.) de Wit & 93 & 155 & 2369,59 & 3,76 \\
Mangifera indica L. & 1 & 2 & 42,41 & 0,17 \\
Manilkara zapota (L.) P. Royen. & 1 & 2 & 51,50 & 0,16 \\
Melaleuca leucadendron L. & 10 & 17 & 59,76 & 0,32 \\
Moringa oleifera Lam. & 8 & 13 & 73,05 & 0,30 \\
Peltophorum pterocarpum (DC) K. Heyne & 4 & 7 & 303,57 & 0,72 \\
Prosopis juliflora (Sw.) DC. & 8 & 13 & 313,24 & 0,50 \\
Tamarindus indica L. & 2 & 3 & 11,32 & 0,05 \\
Terminalia catappa L. & 2 & 3 & 54,04 & 0,07 \\
Terminalia mantaly H. Perrier. & 1 & 2 & 82,47 & 0,19 \\
Total & $\mathbf{1 9 3}$ & $\mathbf{3 2 2}$ & $\mathbf{5 5 2 1 , 1 4}$ & $\mathbf{1 1 , 7 7}$ \\
\hline & & & &
\end{tabular}

Rec. : Recouvrement ; Dens. : Densité ; Surf. Ter. : Surface terrière.

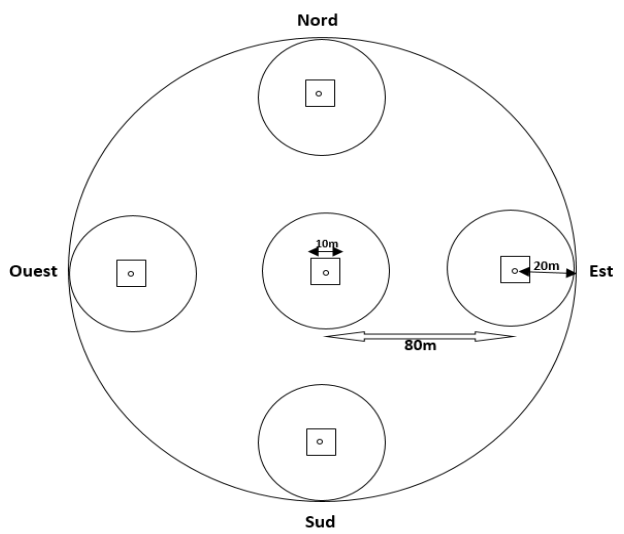

Figure 2: Dispositif d'échantillonnage (grappe de placettes). 


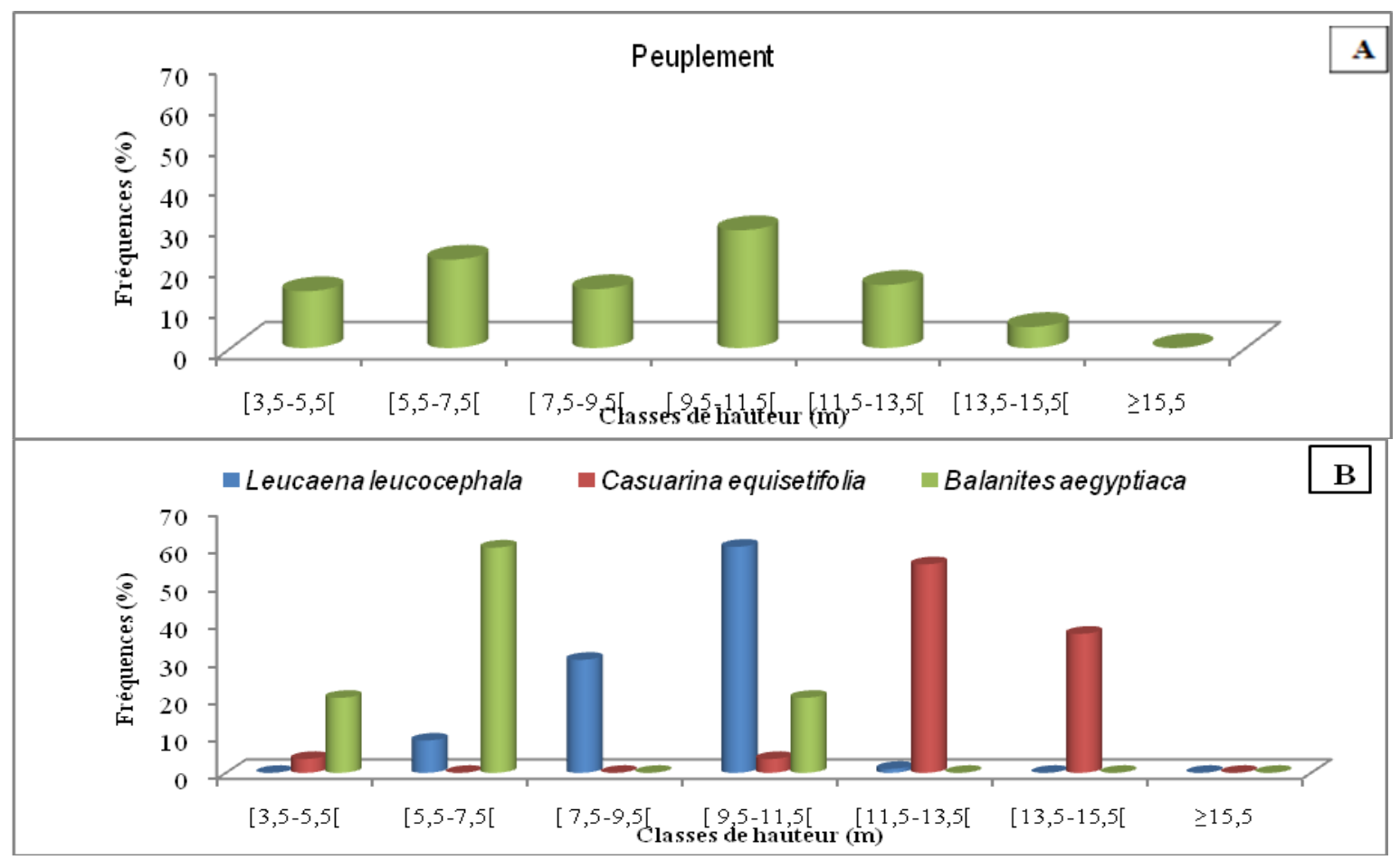

Figure 3: Structure du peuplement et des populations des 3 espèces les plus fréquentes selon la hauteur.

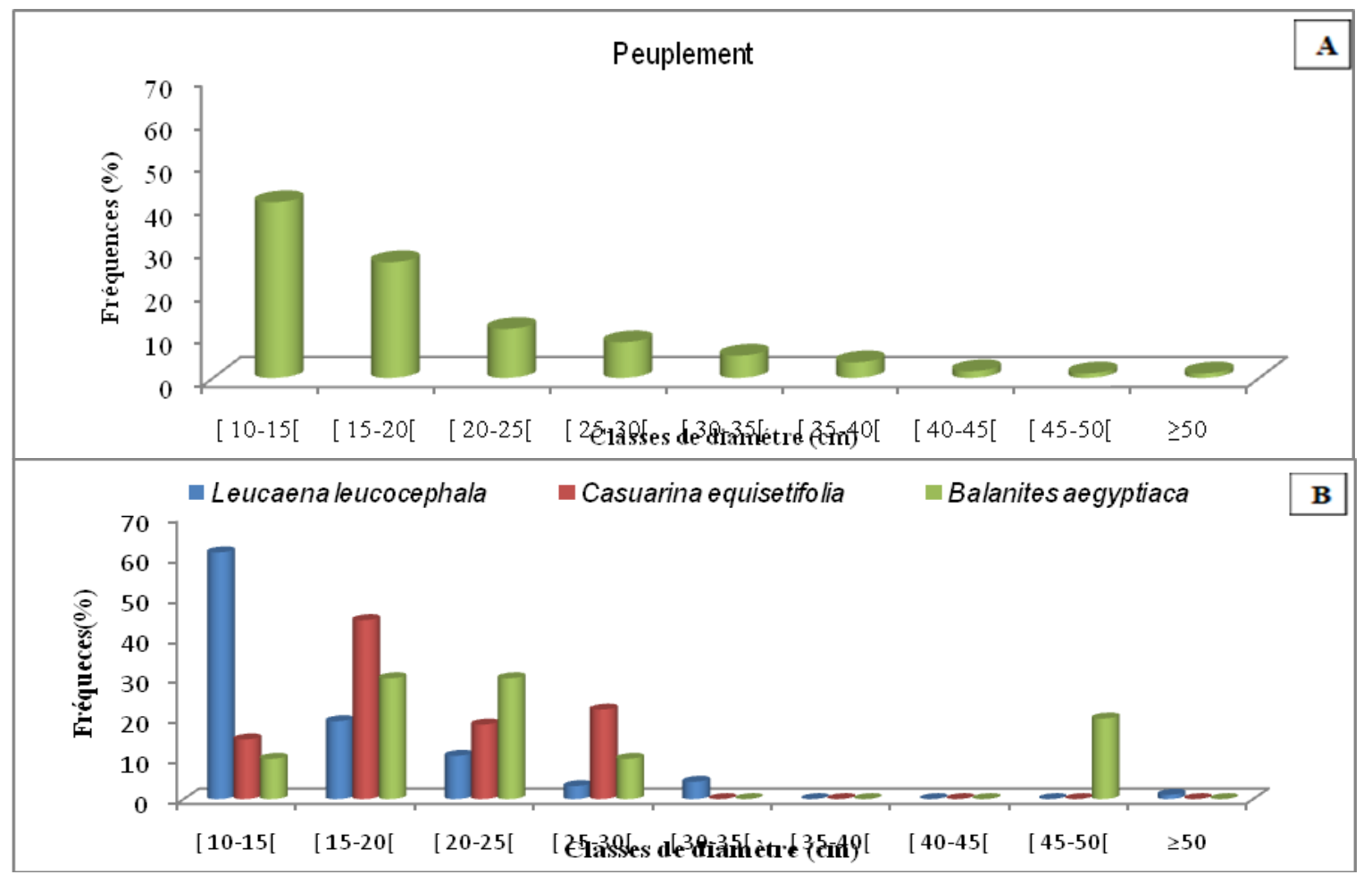

Figure 4:Structure du peuplement et des populations des 3 espèces les plus fréquentes selon le diamètre. 
R. D. DIOP et al. / Int. J. Biol. Chem. Sci. 13(2): 1064-1081, 2019

Tableau 3 : Les valeurs propres et les inerties en pourcentage des cinq premiers axes de l'AFC des espèces ligneuses.

\begin{tabular}{lcccc}
\hline Axes & F1 & F2 & F3 & F4 \\
\hline Valeur propre (bits) & 0,10 & 0,08 & 0,06 & 0,04 \\
Inertie (\%) & 37,01 & 27,95 & 20,35 & 14,70 \\
Cumulé (\%) & 37,01 & 64,96 & 85,30 & 100,00 \\
\hline
\end{tabular}

Points-lignes et Points-colonnes (axes F1 et F2 : 64,96\%)

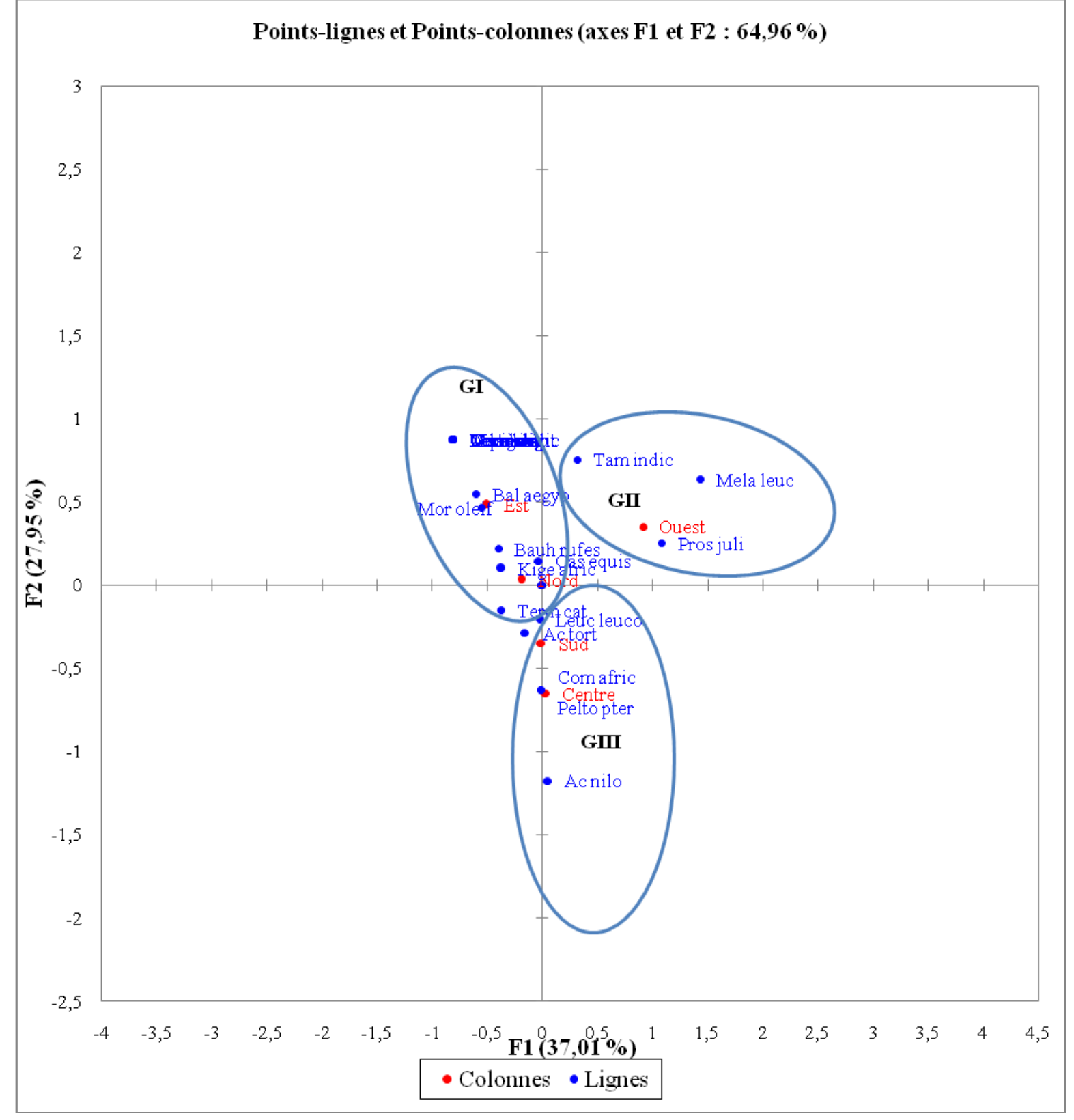

Figure 5 : Diagramme d'AFC des relevés et espèces ligneuses du conservatoire botanique Michel Adanson. 


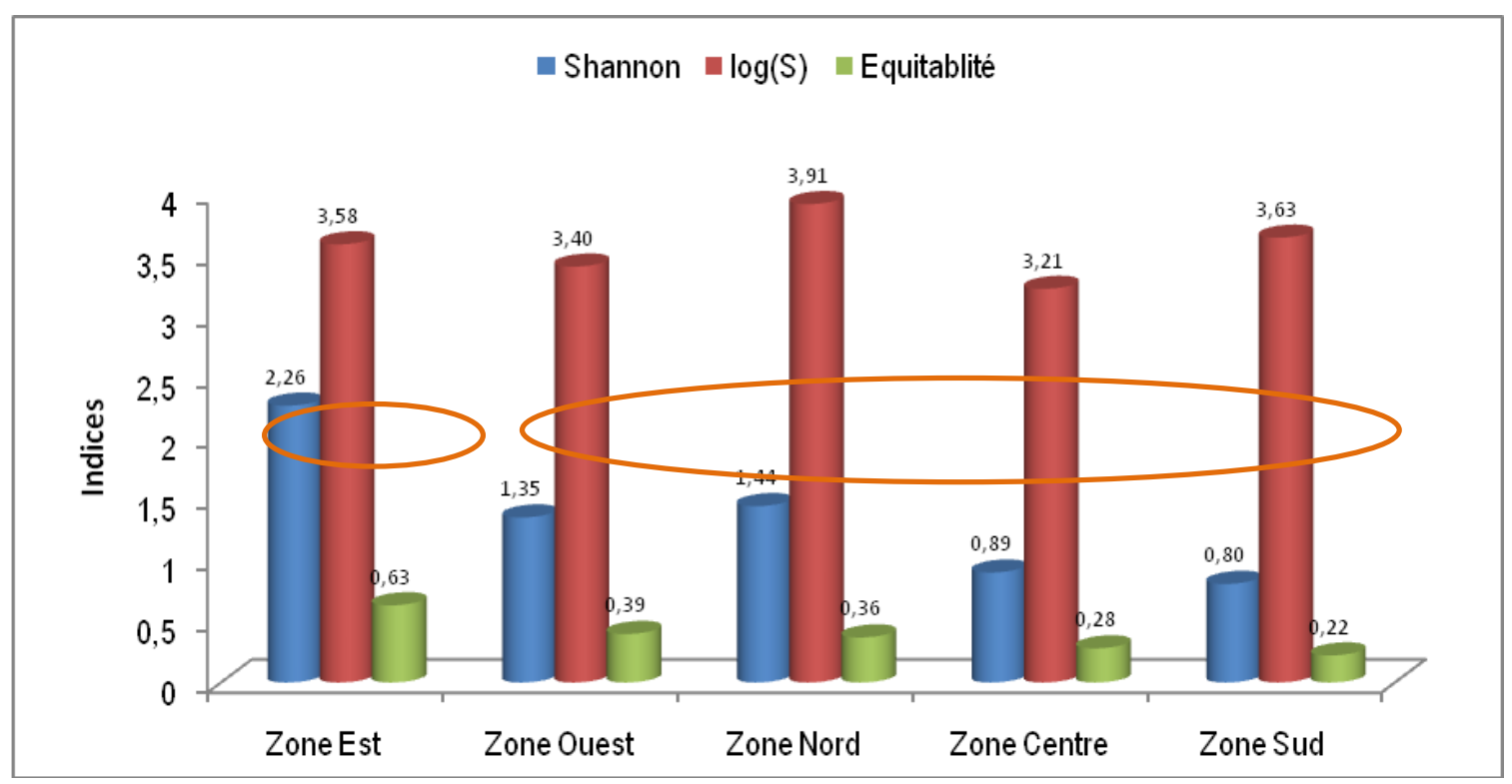

Figure 6 : Répartition des indices de diversité biologique par secteur.

Tableau 4 : Effectif et indice spécifique de régénération des jeunes plants recensés dans le conservatoire.

\begin{tabular}{lcc}
\hline Espèces & $\begin{array}{c}\text { Effectif des } \\
\text { jeunes plants }\end{array}$ & $\begin{array}{c}\text { Indice spécifique de } \\
\text { régénération (ISR) }\end{array}$ \\
\hline Leucaena leucocephala (Lam.) De Wit & 93 & 50,82 \\
Maytenus senegalensis (Lam.) Exell. & 17 & 9,29 \\
Azadirachta indica A. Juss. & 15 & 8,20 \\
Balanites aegyptiaca (L.) Del. & 11 & 6,01 \\
Melaleuca leucadendron L. & 10 & 5,46 \\
Bauhinia rufescens Lam. & 7 & 3,83 \\
Combretum aculeatum Vent. & 5 & 2,73 \\
Guaiacum officinale L. & 5 & 2,73 \\
Grewiabicolor Juss. & 4 & 2,19 \\
Zizyphus mauritiana Juss. & 4 & 2,19 \\
Casuarina equisetifolia Forst. & 3 & 1,64 \\
Kigelia africana (Lam.) Benth & 2 & 1,09 \\
Moringa ovalifolia Dinter et A. Berger & 2 & 1,09 \\
Lepisanthes senegalensis (Juss. ex Poiret) & 1 & 0,55 \\
Crataeva adansonii DC. & 1 & 0,55 \\
Guazuma ulmifolia Lam. & 1 & 0,55 \\
Phyllenthus acidus (L.) Skeel. & 1 & 0,55 \\
Adansonia digitata L. & 1 & 0,55 \\
TOTAL & $\mathbf{1 8 3}$ & $\mathbf{1 0 0 , 0 0}$ \\
\hline
\end{tabular}




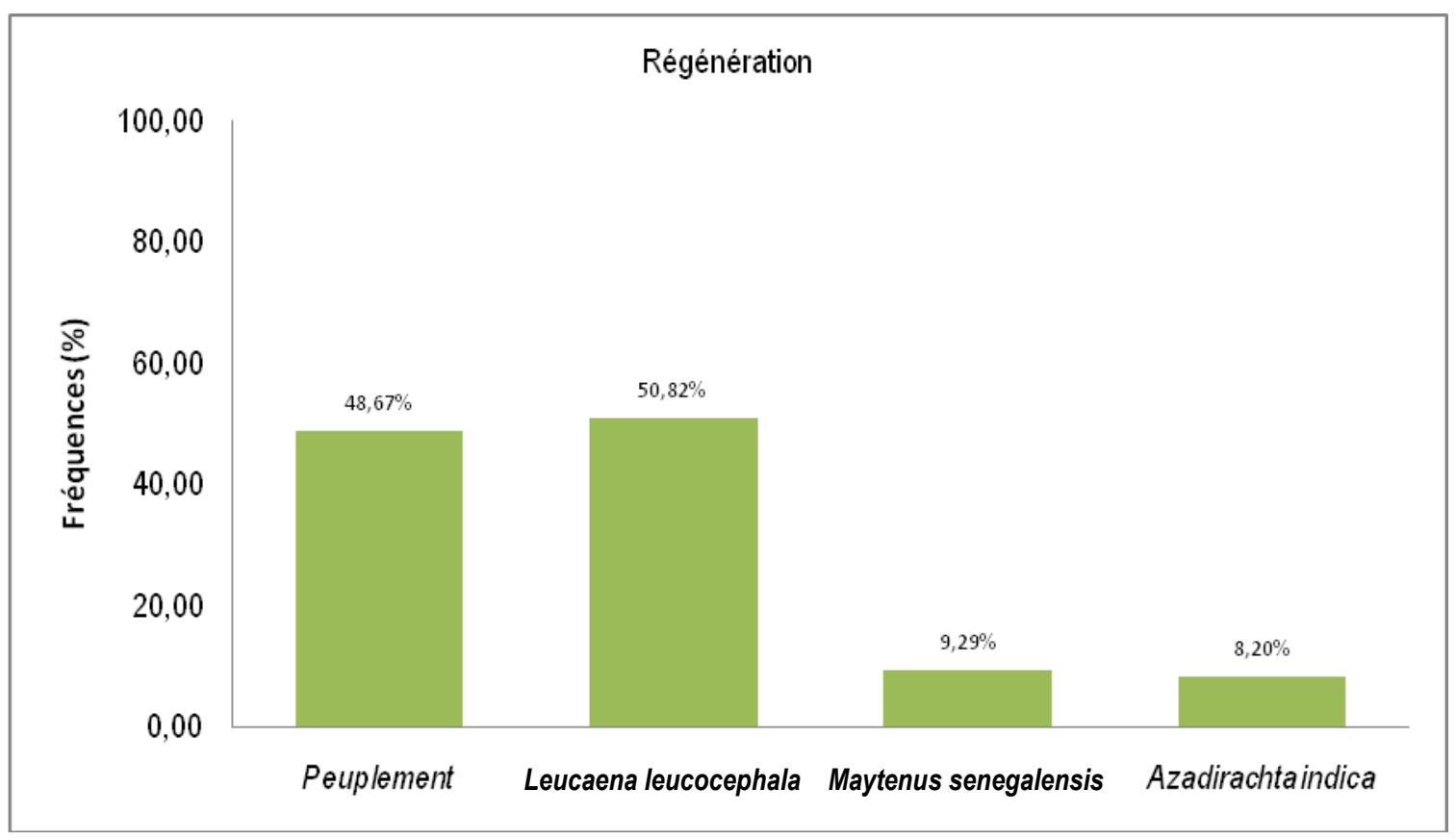

Figure 7: Régénération du peuplement et l'indice spécifique de régénération des trois espèces les plus fréquentes.

\section{DISCUSSION}

Dans l'unité de végétation échantillonnée, le peuplement ligneux est riche de 23 espèces réparties dans 21 genres et 16 familles. Dans la végétation, une densité, une surface terrière et un recouvrement aérien assez élevées ont été observés. La distance moyenne entre individus est faible, et un coefficient de variation élevé suggérant ainsi une distribution en bosquets (Diouf, 2000 ; Diatta et al., 2009). Comparée à la végétation de la réserve naturelle de Ngazobil, celle du conservatoire botanique Michel Adanson présente des proportions plus faibles au niveau de la densité et de la surface terrière. La densité la végétation du CBMA est de 322 individus/ha contre 1464 individu/ha pour celle de la réserve naturelle de Ngazobil. Il en est de même pour la surface terrière qui est de $11,77 \mathrm{~m}^{2} /$ ha pour le CBMA contre $59,63 \mathrm{~m}^{2} / \mathrm{ha}$ pour la RNN.

La végétation est à la fois arborée et arbustive contrairement à celle de la réserve de Ngazobil qui est seulement arbustive (Diatta et al., 2009). Leucaena leucocephala (100\%), Prosopis juliflora (60\%), Kigelia africana $(60 \%)$ y sont les espèces dominantes et aussi les plus fréquentes; ce sont des espèces à port arboré et arbustive. La forte densité de Leucaena leucocephala nous pousse à parler de la «leucaenisation» de la végétation du conservatoire botanique Michel Adanson.

La structure des ligneux suivant la hauteur nous a permis de distinguer deux strates : une arbustive à Balanites aegyptiaca et une arborescente à leucaena leucocephala et Casuarina equisetifolia.

La structure des ligneux suivant le diamètre montre un modèle de distribution type exponentiel décroissant ou structure en «L». D’après Sambou (2004), cette structure est caractérisée sur la base de la répartition des individus en classe de diamètre, par un grand nombre de petits individus, un petit nombre de grands individus et une réduction régulière du nombre d'individus d'une classe de dimension à la suivante. Les structures en «L des peuplements des différents groupements ligneux décrits traduisent des formations stables (Ouédraogo, 2006). L'équilibre de ce peuplement ligneux révèle 
de la capacité d'adaptation aux pressions du milieu, notamment les feux précoces ainsi que les conditions édaphiques (Ouedraogo et al., 2008).

L'AFC nous a permis d'apprécier la distribution des espèces ligneuses dans le conservatoire. Cette distribution est très hétérogène et est divisée en trois groupes. Le groupe 1 qui se trouve à l'Est du site et qui inclus l'ISED a reçu le plus des plantes différentes pour la conservation. Ceci s'explique par le fait qu'elle est la zone la plus accessible aux visiteurs et par conséquent elle bénéficie de plus d'entretien de part des responsables du site. Quant au groupe 2 à l'Ouest du conservatoire referme des groupements de Prosopis qui forment des haies qui délimitent certaines parcelles dans lesquelles sont plantées des espèces comme Melaleuca leucadendron et Tamarindus indica. Il constitue la zone la moins diversifiée du conservatoire. Les prochaines introductions de plantes doivent se faire dans cette zone afin d'équilibrer la répartition de la diversité végétale dans le site. Le groupe 3 concerne les espèces plantées au milieu et au Sud du conservatoire avec plus de diversité végétale vers le Sud.

Le calcul d'indice d'équitabilité de Piélou et d'indice de Shannon montre que la flore ligneuse $\mathrm{du}$ conservatoire botanique Michel Adanson de Mbour est caractérisée par une diversité moyenne $(\mathrm{H}=2)$ et une distribution hétérogène des espèces dans le milieu (J proche de 0 ). Cette végétation est caractérisée par la dominance d'un petit nombre d'espèce à forte abondance. C'est le cas de Leucaena leucoceplela, Melaleuca lecadendron et Balanites aegyptiaca. Cependant le secteur Ouest est un peu différent des autres, car c'est une zone à diversité plus importante ou la plupart des espèces est représentée par un seul pied. Cette zone est dominée par le Niaouli (Melaleuca lecadendron). Cette inégalité s'explique par le fait que le secteur Ouest abrite l'ISED un parc des plantes ligneuses sélectionnées et conservée en fonction de leur valeur médicinale et/ou ornementale. Ces plantes très diversifiées sont représentées pour la plupart par un seul pied. Selon Djego et al. (2012), une plus grande diversité implique une plus grande égalité des contributions individuelles. Inversement, une diversité plus faible signifie une faible régularité de la répartition des individus. Dans les zones Sud, Centre, Nord et Ouest, la diversité biologique est très faible malgré le nombre d'individus élevé. La dominance du leucaena leucoceplela est plus accentuée dans la zone Nord suivit de la zone Centre et enfin la zone Sud. Cette plante a été utilisée pour former la haie de séparation de plusieurs parcelles dans ces zones. Comparée à la réserve naturelle de Ngazobil, Diatta et al. (2009) avait montré que le peuplement ligneux de cette végétation était assez homogène, donc identique avec celle du conservatoire botanique Michel Adanson. Ceci s'expliquerait par la dominance particulière d'une seule strate et une seule espèce ou de manque d'une grande variabilité écologique de la réserve qui se trouve en fait sur une surface topographique plane, avec des pentes relativement faibles vers la mer (Diatta et al., 2009) tout comme le conservatoire Michel Adanson.

Le terme régénération naturelle englobe toutes les stratégies utilisées par les plantes pour établir leurs stades juvéniles. Elle est importante non pas seulement pour cette fonction, mais assure également le remplacement de chacun des membres d'une communauté qui meurt au terme de son cycle de vie (Baraloto, 2003). La structure par classes de diamètre a montré que le peuplement régénère bien par la forte représentation des classes inférieures de diamètre. Selon Badji et al. (2014), la prédominance d'arbres de petits diamètres traduit une capacité de régénération du peuplement. Ces résultats sont identiques avec ceux trouvés par Diatta et al. (2009) dans la réserve naturelle de Ngasobil. D'après Kebenzikato et al. (2014), un peuplement où il y aurait abondance d'individus à faibles diamètres est un peuplement en pleine régénération tandis qu'un peuplement où il $\mathrm{y}$ aurait beaucoup d'individus à grande circonférence est un peuplement vieillissant. La densité et le taux de régénération élevés de 
leucaena leucocephala pourraient être liés à des conditions favorables et à une bonne capacité de dissémination et de germination de la graine.

Les individus de grande taille et de faible diamètre constituent l'essentiel du peuplement ligneux. Une telle distribution met aussi en évidence une compétition pour la lumière et une manque d'exploitation des plantes du conservatoire qui sont protégées des actions anthropiques.

La présence d'individus proches les uns des autres montres que la végétation se développe dans des conditions climatiques favorables avec le système d'arrosage goutte à goutte qui empêche le manque d'eau en saison sèche.

\section{Conclusion}

Ce travail qui avait comme objectif de déterminer la structure de la végétation du conservatoire botanique Michel Adanson a donné plusieurs résultats. L'étude de la végétation ligneuse du conservatoire botanique Michel Adanson a permis d'inventorier 193 individus réparties dans 23 espèces, 21 genres et 16 familles. La végétation à la fois arborée et arbustive est caractérisée par une densité, une surface terrière et un recouvrement aérien assez élevés. Cette végétation est dominée par des espèces comme Leucaena leucocephala, Prosopis juliflora et Kigelia africana. Ces espèces sont diversement reparties dans le site. Le peuplement ligneux est dominé par des individus de faible diamètre et de taille haute dû à la forte compétition vis-à-vis des ressources du milieu. Il est caractérisé, dans l'ensemble, par une diversité moyenne et une distribution hétérogène des individus dans les parcelles. Prise par secteur, la zone Est a la diversité biologique la plus élevée avec une répartition assez homogène des individus, tandis que les zones Sud, Centre, Nord et Ouest ont une faible diversité spécifique avec une distribution hétérogène des individus malgré leur nombre élevé. Le peuplement régénère bien par la forte représentation des classes de diamètre inférieures. Une gestion durable des ressources végétales du CBMA nécessite une connaissance de la végétation.

\section{CONFLIT D'INTERETS}

Les auteurs déclarent qu'il n'y aucun conflit d'intérêts pour cette article.

\section{CONTRIBUTIONS DES AUTEURS}

RDD a collecté, traité et analysé les données puis rédigé et corrigé l'article. MSM, $\mathrm{CB}$, ID ont contribué à la rédaction et correction de cet article. OS a contribué à la mise en place du dispositif de collecte des données et à la correction du document. AAC a contribué l'identification des espèces et à la réactualisation des noms scientifiques. MTAS, $\mathrm{KN}$ ont contribué à la rédaction et à la correction de l'article.

\section{REMERCIEMENTS}

Les auteurs remercient ENDAMADESAHEL qui, à travers l'assistance financière et la mise à disposition du conservatoire botanique Michel Adanson de Mbour, a facilité la réalisation de ce travail.

\section{REFERENCES}

Adjakpa JB, Yedomonhan $\mathrm{H}$, Ahoton LE, Weesie PDM, Akpo LE. 2013. Structure et diversité floristique des îlots de forets riveraines communautaires de la vallée de Sô du Benin. Journal of Applied Biosciences, 65: 4902-4913. DOI: https://www.ajol.info/index.php/jab/artic le/view/89573.

Badji M, Sanogo D, Akpo LE. 2014. Dynamique de la végétation ligneuse des espaces sylvo-pastoraux villageois mis en défens dans le Sud du Bassin arachidier au Sénégal. Bois et forêts des tropiques, 319(1). DOI: bft.cirad.fr/cd/BFT_319_43-52.pdf

Badji M, Sanogo D, Akpo LE. 2013. Effet de l'âge de la mise en défens sur la reconstitution de la végétation ligneuse des espaces sylvo pastoraux du sud bassin arachidier (Sénégal). Journal of Applied Biosciences, 64: 4876-4887. DOI: 
http://www.m.elewa.org/JABS/2013/64/ 12.pdf

Baraloto C. 2003. Régénération forestière naturelle : de la graine à la jeune tige. Rev. For. Fr. $L V$ - numéro spécial.

Cornier T, Toussaint B, Duhamel F, Blondel C, Henry E, Mora F. 2011. Guide pour l'utilisation d'arbres et d'arbustes pour la végétalisation à vocation écologique et paysagère en Région Nord-Pas de Calais. Centre régional de phytosociologie / Conservatoire botanique national de Bailleul, pour le Conseil régional Nord, Calais et la DREAL, 48p.

Crow TR, Haney A, Waller DM. 1994. Report on the scientific round table on biological diversity convened by the chequamegon and nicolet National forest. General technical report NC- 166, USA Forest service, North Central forest Experiment Station, Saint PaulMinnesota, USA.

Dessey N. 2006. Dynamique de la végétation et du climat : étude par télédétection de cinq biomes brésiliens, forêt ombrophile dense et ouverte, cerrados, caatinga et campanhagaúcha. Thèse de doctorat, Université Paris X, Nanterre, 362p.

Diatta CD, Gueye M, Koma S, Akpo LE. 2009. Diversité de la flore et de la végétation ligneuses de la réserve de Ngazobil (Joal-Fadiouth) au Sénégal. Journal des Sciences, 9(3): 1-13.

Diouf M. 2000. Dynamique des écosystèmes sahéliens : Effets des microsites topographiques sur la diversité de la végétation ligneuse au Ferlo (Nord Sénégal). Mémoire de DEA, Faculté des Sciences et Techniques, Université Cheikh Anta Diop, Sénégal, 44p.

Djego J, Gibigaye M, Tente B, Brice Sinsin B. 2012. Analyses écologique et structurale de la forêt communautaire de Kaodji au Bénin. Int. J. Biol. Chem. Sci., 6(2): 705713. DOI

https://www.ajol.info/index.php/ijbcs/arti cle/view/80618/70859.
Frontier S, Pichod-Viale D. 1995. Ecosystèmes : $\quad$ Structure, Fonctionnement, Evolution (2Ed). Collection d'écologie: Paris-Masson; 21: 287-311.

Henry E, Cornier T, Toussaint B, Duhamel F, Blondel C. 2011. Guide pour l'utilisation de plantes herbacées pour la végétalisation à vocation écologique et paysagère en Région Nord-Pas de Calais. Centre régional de phytosociologie / Conservatoire botanique national de Bailleul, pour le Conseil régional Nord, Calais et la DREAL, 56p.

Kebenzikato AB, Wala K, Dourma M, Atakpama W, Dimobe K, Pereki $\mathrm{H}$, Batawila K, Akpagana K. 2014. Distribution et structure des parcs à Adansonia d igitata L. (baobab) au Togo (Afrique de l'Ouest). Afrique SCIENCE, 10(2): 434-449.

Legendre L, Legendre P. 1984. Ecologie numérique. In La Structure des Données Ecologiques (Tome 2). Masson collection d'écologie. DOI: https://onlinelibrary.wiley.com/doi/abs/1 0.1002/iroh.19810660515.

Magurran AE. 2005. Measuring Biological Diversity. Wiley-Blackwell; 264p.

Mballo M. 2010. Inventaire taxonomique et importance médicinale des plantes des secteurs 13 à 14 du conservatoire botanique Michel Adanson de Mbour. Mémoire de fin d'études d'ingénieur des travaux des eaux et foret, Institut de formation agricole (ISFAR), Université de Thiès, $82 \mathrm{p}$.

Ouédraogo A. 2006. Diversité et dynamique de la végétation ligneuse de la partie orientale du Burkina Faso. Thèse de doctorat, Université de Ouagadougou, $196 \mathrm{p}$.

Ouedraogo O, Thiombiano A, Hahn-Hadjali K, Guinko S. 2008. Diversité et structure des groupements ligneux du parc national d'Arly (Est du Burkina Faso). Flora et Vegetatio Sudano-Sambesica, 11: 5-16. 
Peet RK. 1974. The Measurement of Species Diversity. Annual Review of Ecology and Systematics, 5(1974): 285-307. DOI: https://www.annualreviews.org/doi/.../an nurev.es.05.110174.0014

Picard N. 2006. Méthode d'inventaire forestier. Projet de développement rural participatif dans le moyen Atlas central (projet Khenifra), 43p.

PNUE. 2012. Global Environment Outlook 5. PNUE, Nairobi.

Poupon H. 1980. Structure et Dynamique de la Strate Ligneuse d'une Steppe Sahélienne au Nord du Sénégal. Orstom éd. : Paris ; 307p.

Roberts-Pichette P, Gillespie L. 2002. Protocoles de suivi de la biodiversité végétale terrestre. Le réseau d'évaluation et de surveillance écologiques, Canada.

Sambou B. 2004. Evaluation de l'Etat, de la dynamique et des tendances évolutives de la flore et de la végétation ligneuses dans les domaines soudanien et subguinéen du Sénégal. Thèse de Doctorat d'Etat, Institut des Sciences de l'Environnement, Université Cheikh AntaDiop, 210p.

Tine M. 2009. Analyse des impacts socioéconomiques et spatiaux du PAPA-SUD dans la Petite Côte ; cas de Mbour et Joal. Mémoire de master, Université Gaston Berger de Saint Louis, 85p.

Tremblay FM, Bergeron Y, Lalond D, Maufette Y. 2002. The sexual effects of potential reproduction and seedling recruitment on the maintenance of red maple (Acer rubrum L.) populations at the northern limit of the species range. Journal of Biogeography, 29: 365-373. DOI: https://onlinelibrary.wiley.com/doi/abs/... /j.1365-2699.2002.00665 